\title{
Self-perception of disability and prospects for employment among U.S. veterans
}

\author{
Christopher L. Griffin, Jr. ${ }^{\text {a,* }}$ and Michael Ashley Stein ${ }^{\mathrm{b}, \mathrm{c},}$ \\ ${ }^{\mathrm{a}}$ William \& Mary Law School, Williamsburg, VA, USA \\ ${ }^{\mathrm{b}}$ Harvard Law School Project on Disability, Cambridge, MA, USA \\ ${ }^{\mathrm{c}}$ Harvard Law School, Cambridge, MA, USA
}

Received 23 May 2013

Accepted 23 October 2013

\begin{abstract}
.
BACKGROUND: Barriers to employment in the civilian labor force are increasingly difficult problems for returning veterans with disabilities. Reduced self-perception of disability status because of predominant military norms can be particularly harmful to reintegration efforts.

OBJECTIVE: We analyze rates of self-identified and externally determined disability status among U.S. veterans. Evidence of a lower self-report rate would confirm the hypothesis that armed forces culture might hold back truly deserving veterans from seeking the benefits owed, including specialized employment training programs.

METHODS: We use data from the Current Population Survey Veterans Supplement over the sample period 1995-2010 on disability status and associated demographic characteristics to present descriptive measures and limited statistical inference.

RESULTS: Over the entire sample period, federal agencies considered $29 \%$ of the survey respondents to have a serviceconnected disability versus a $9 \%$ self-identification rate. The rate of more severe service-connected disabilities has risen steadily, while less drastic disability rates have fallen. Non-white respondents and those with lower education levels were less likely to self-identify.

CONCLUSIONS: Large disparities in internal and external disability status identification raise questions about targeting soldiers re-entering the labor force. Employment policy should focus on overcoming negative cultural stereotypes and encouraging selfidentification.
\end{abstract}

Keywords: Labor force, disabled soldiers, self-identification

\section{Introduction}

Societies across boundaries and time have felt a duty to their veterans with disabilities, and have devised programs to provide them with benefits (whether social welfare or pensions), and when feasible, to reintegrate these individuals back into society [1]. One of the chief goals of these assimilation schemes has been

*Corresponding author: Christopher L. Griffin, Jr., William \& Mary Law School, P.O. Box 8795, Williamsburg, VA 23187, USA. Tel.: +1 757221 1251; Fax: +1 757221 3261; E-mail: clgriffinjr@ wm.edu. renewed employment. Our study is underscored by the notion that labor force attachment provides the quickest means for social incorporation and provides injured military personnel the means by which to secure both financial autonomy and community participation. As noted by Cohen [2], the success of these ventures has been highly contextual and dependent on broader cultural factors. What remains constant, however, is an effort by nations to reincorporate their wounded veterans and to treat them preferentially relative to those individuals whose disabilities did not arise in the course of military activity. This is equally true for societies as diverse as democratic Israel [3] and post-revolutionary Iran [4] 
In determining the suitability of individuals for veteran-specific disability schemes, governments have sought to draw a line on eligibility between those "truly" disabled and others who may have suffered impairments but not of a level commensurate with a duty to expend public resources. Although states attempt to empirically distinguish these two groups, available evidence indicates that social constructions of disability as well as racial, ethnic, and other biases - skew those attempts. This effect is clearly documented by a series of historical research projects by Blanck and colleagues $[5,6]$ on the implications of administrative personnel assessing the severity of Union solider injuries incurred during the Civil War and their worthiness for government sponsored pensions. The influence is likewise documented by contemporary research evaluating appraisals of disabled American veterans in the postIraq/Afghanistan era [7, pp. 88-89].

Complicating this situation is a military culture that emphasizes the physical and mental well-being of soldiers, as well as their independence and selfreliance [8]. That overarching milieu is understandable in view of the activities and circumstances in which military personnel engage. Nevertheless, "Warrior Culture" ultimately lends itself to injured soldiers initially downplaying the extent or long-term natures of their disabilities [9]. Indeed, anecdotal evidence that one of the authors has learned through the National Organization on Disability's Wounded Warriors program indicates that veterans heavily resist the label of "disabled" and instead, often in the face of contrary and graphic evidence, classify themselves as "injured" or "wounded." Refusal to accept disability status leads both to delay in seeking disability-specific benefits and also stymies the growth of a potentially powerful political movement in which persons with disabilities, whether or not their disabilities originate in armed activities, coalesce around areas of mutual concern [10].

This paper explores the tension between self-identification and external verification of disability among contemporary American veterans with disabilities, and how varying affirmations of disability status by certain demographic characteristics might impact available support services, benefits, and life choices of disabled veterans. (Consistent with our analytical objectives, we treat self-reports and administrative determinations as indicative of a physical or mental servicerelated impairment rather than use a formal definition of disability.) To do so we chart the post-combat identification of veterans with disabilities from 1995 to 2010 , both through their own responses to interview- ers and through government agency certifications. Our research suggests that self-reports of disability status, although increasing slightly over time, lag far behind government agencies' determinations. Using two consistently captured measures of disability status in our data, around 4-5\% of veterans report having a disability over the sample period. The Department of Veterans Affairs (VA) and Department of Defense (DoD) on the other hand reached conclusions indicating over twice the rate of service-connected disabilities (10-15\%).

The empirical analysis that follows describes only the incidence of disability self-reports in addition to external designations over time and according to observable veteran characteristics. Where applicable, we test for statistically significant differences in disability measures according to those observables. Otherwise, we stop short of discussing broader statistical correlations and certainly causal pathways. Making stronger claims about the causes and consequences of veterans' disabilities requires richer data and a research design beyond the scope of this paper. Our findings nevertheless advance the conversation about how best to address the underemployment of veterans with disabilities. Although we cannot connect these underreports of disability status to specific military cultural norms, we believe stigma over self-identifying as disabled - especially among male service members - probably explains a good portion of the disparity observed. Overcoming these impediments will be a necessary precursor to enhancing employment opportunities for men and women returning from service.

The paper proceeds as follows. Section 2 provides additional background on the friction between servicerelated disabilities and military cultural norms. Section 3 describes the data source and our descriptive findings. Section 4 returns to the question of how self-perceptions of disability impact job training and employment opportunities. We conclude with some thoughts on future research.

\section{Background}

The optimal approach to reducing under- and unemployment among U.S. veterans is a decidedly empirical issue. Philanthropic organizations and legislators have proposed additional funding for training and reintegration programs as solutions. These innovations at best would supplement first-order mental and physical treatment for trauma sustained on the battlefield. Monetary support should figure prominently in a re- 
Table 1

Summary statistics

\begin{tabular}{lccc}
\hline Variable & $N$ & Mean & SD \\
\hline Self-reported disability (labor force recode) & 91,192 & $4.9 \%$ & $21.6 \%$ \\
Self-reported disability (work last week) & 91,192 & $3.8 \%$ & $19.1 \%$ \\
VA/DoD reported disability & 91,192 & $11.8 \%$ & $32.3 \%$ \\
Age & 91,192 & 58.9 & 15.9 \\
Male & 91,192 & $94.6 \%$ & $22.6 \%$ \\
White & 91,192 & $89.5 \%$ & $30.7 \%$ \\
Education & & & \\
$\quad$ Less than high school & 91,192 & $10.6 \%$ & $30.7 \%$ \\
Less than a bachelor's degree & 91,192 & $64.4 \%$ & $47.9 \%$ \\
At least a bachelor's degree & 91,192 & $25.1 \%$ & $43.3 \%$ \\
\hline
\end{tabular}

Source: CPS Veterans Supplement.

vised strategy for sustaining veteran re-entry, but those resources will be more effective the more completely we understand that such problems cannot be fixed with money alone.

Attending to veterans with disabilities requires that we first understand the norms of military attitudes regarding disability. Military culture is "infused during basic training" and reinforced throughout one's career [9, p. 509]. It emphasizes "strength, resilience, [and] courage." Immersion in this culture leads soldiers who are no longer able to contribute to their unit's collective effort due to disabling conditions to have "feelings of failure, weakness, and guilt" [11, p. 9A]. The prevailing "warrior mentality," instead, commands that injured service personnel should "'man up' and 'get off their butts"' [12, p. C01]. Consequently, the physical and psychological impact of disablement on active military personnel can be profound [13]. One reason proffered is that impairment could threaten the trust that each unit member has in the integrity of the entire unit. If a soldier is not "battle ready" after a disabling event, some might worry about the safety of his or her cohorts.

Historically, military veterans with mental health problems have been especially stigmatized and made to feel their conditions were either insignificant or of their own making. Contributing to this dynamic are diagnoses that both label a particular medical pathology and signal an unmilitary-like social condition such as "soldier's heart" or "shell shock" [14, pp. 789794]. One result of this schema is the internalization, by injured service members, of perceptions of inadequacy. A study [15, pp. 13-22] of several thousand psychologically injured Army and Marine Corps veterans from Afghanistan and Iraq, for example, found that they did not disclose their mental health conditions because they feared stigma and repercussions. Specifically, 65\% believed they "would be seen as weak"; $63 \%$ felt that their "unit leadership would treat [them] differently"; and 59\% were concerned that "members of [their] unit might have less confidence in [them]" [16, p. 3A]. A second manifestation of this prevailing military culture that discounts the veracity or significance of mental health trauma is reluctance among injured service personnel themselves to seek diagnosis and treatment. As stated by the chief of mental health at one Veteran's Administration hospital, "it's an ongoing frustration to try to get veterans to seek help" [17].

\section{Data analysis}

The Bureau of Labor Statistics and the Census Bureau produce the Current Population Survey (CPS), a standard dataset for economists studying national trends in employment levels, wage growth, and household characteristics. Although the CPS data contain rich information on labor market outcomes and are designed primarily to facilitate traditional economic analysis, scholars [18,19] have used the survey to study Americans with disabilities. We have documented the perils of using the CPS for these purposes elsewhere [20], namely that the variable from which disability status is measured bears only a passing resemblance to our current social or legal understandings of the term. Thankfully, the once biennial, now annual, Veterans Supplement to the main CPS questionnaire provides several checkpoints that make identifying persons with disabilities arguably more reliable. As discussed below, we also can compare survey responses with an outside status assessment through the VA/DoD rating system.

We collected from each biennial dataset during the period 1995-2009 and again in 2010 select variables related to demographic characteristics, service histo- 
ries, and disability status. Summary statistics for the working dataset of about 91,000 respondents appear in Table 1. This subset of the entire CPS supplement excludes non-veterans, identified through a question about when the respondent served in the armed forces. The remaining former service members selfidentified as having a disability between $4-5 \%$ of the time, whereas the mean VA/DoD determination rate was about $12 \%$ over the same period. Sampled veterans were predominantly white men aged about 60 years old, and almost two-thirds possessed at least a high school diploma but not a four-year college degree. Although the age profile is skewed toward the higher end of the distribution, returning soldiers from the Second Iraq War and Afghanistan were sampled. Thus, we are confident that the statistics reported in this paper are at least partially reflective of recent veteran experiences. A few other variables dealing with aspects of service are not reported in Table 1 because their means are more difficult to report straightforwardly. For these metrics, however, we mention that mean VA/DoD disability ratings fell near a category containing all ratings from $30-49 \%$; that veterans had been separated from the armed forces on average around 1968; and that, in total, they served about 3-4 years by the time of their interviews.

Three disability measures among a few other possibilities both showed up in each year's questionnaire and contained enough non-missing values to be useful. These variables reflect decidedly different conceptions of disability in increasing order of precision. First, answers to other labor force questions in the dataset could, when taken together, allow the interviewer to infer that a respondent was not in the labor force because of a disability. Although this variable might resemble the external VA/DoD determination in form (because one is inferring status from circumstantial evidence), its substantive values are still dependent on respondent answers. Second, interviewers queried: "Last week, did you do any work for either pay or profit?" One response indicates that the interviewee was disabled. This question arguably is preferable to the previous one as a self-report measure in that one's disability status is triggered directly by a question rather than inferred through a recoding process. Yet, like the main CPS questionnaire, self-reports of disability often do not track either the administrative agency's conclusion or state/federal legislation's definitions. Finally, the external measure - whether the VA or DoD has determined that the respondent has a service-connected disability - extends the previous self-report measures in a way precluded by the main CPS questionnaire and other labor economics datasets. Specifically, we are able not only to perform cross-checks between agency findings and respondents' own understandings of their mental and physical conditions but also to investigate any disparities between and trends across the two assessments.

Answers to the VA/DoD question admittedly come from respondents themselves rather than directly from the agency; thus, respondents might deliberately or unconsciously reply with bias in either direction. For example, if a veteran did not consider her/himself to have a qualifying disability, he or she might answer the VA/DoD question negatively even though an agency has determined that he or she has a service-connected disability. Or the same respondent might respond affirmatively to the same question even though neither agency found a disability because she believes that she has one. Without independent verification from VA/DoD it is impossible to establish the incidence and degree of such bias. For our limited, descriptive purposes we simply assume that answers are equally likely to be biased in either direction and therefore have no net effect.

As Figs 1 and 2 show, self-reports of disability were fairly stable through the late 1990s at about 3-4\% and then started to increase in the 2000s. Interestingly, these trends coincided with a decline in the number of veterans represented in the biennial CPS supplement from 11,569 in 1999 to 9739 in 2010 (-16\%). One likely explanation could be that veterans were returning from Iraq and Afghanistan exactly when the growth in disability reports emerges. In addition, we observe scale differences between the two self-report measures. Disability reports were slightly more likely each year when gleaned from interviewer recoding instead of a direct response about working in the previous week. The difference might be attributable to short-term disabilities that kept the respondent from working in the last week but that did not remove him/her from the labor force entirely. Still, one might expect that direct questions are more likely to capture affirmative answers than interviewer inference.

Another cut at these data, however, confirms that the recoding measure reliably tracks underlying selfconceptions of disability status. A cross-tabulation of the two variables demonstrates, for example, that self-reports of another employment status (retirement) overwhelmingly correspond to recodes of the same (96\%). Similarly, $84 \%$ of persons self-reporting a disability are denoted as such by interviewers. We there- 


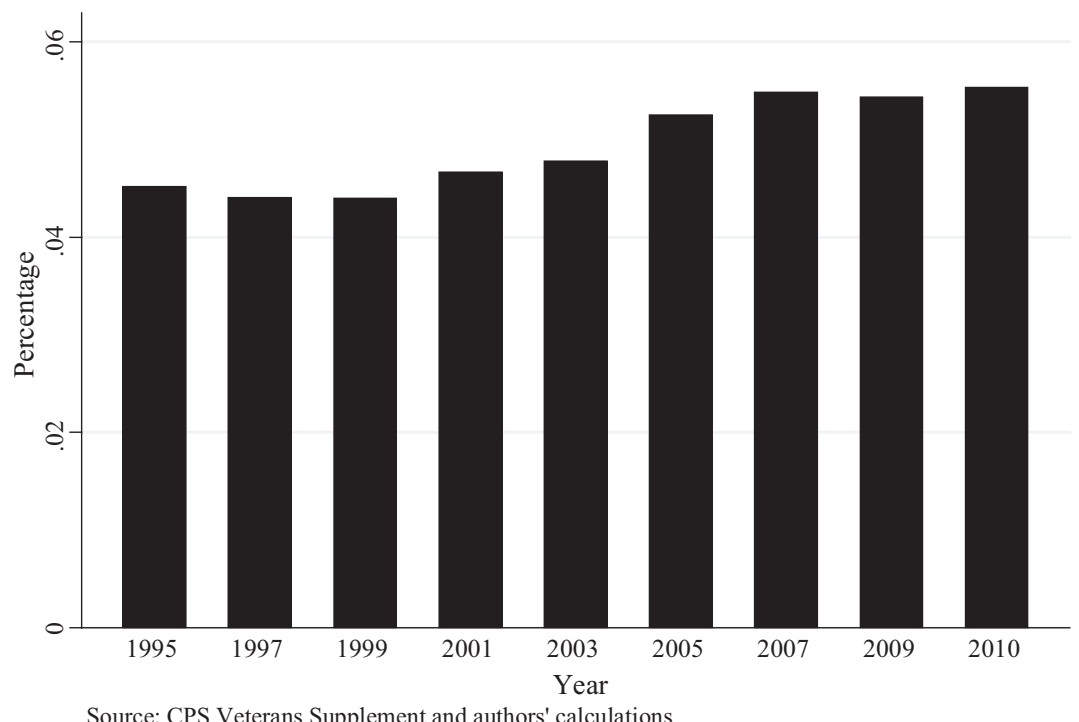

Fig. 1. Self-report of disability using labor force question.

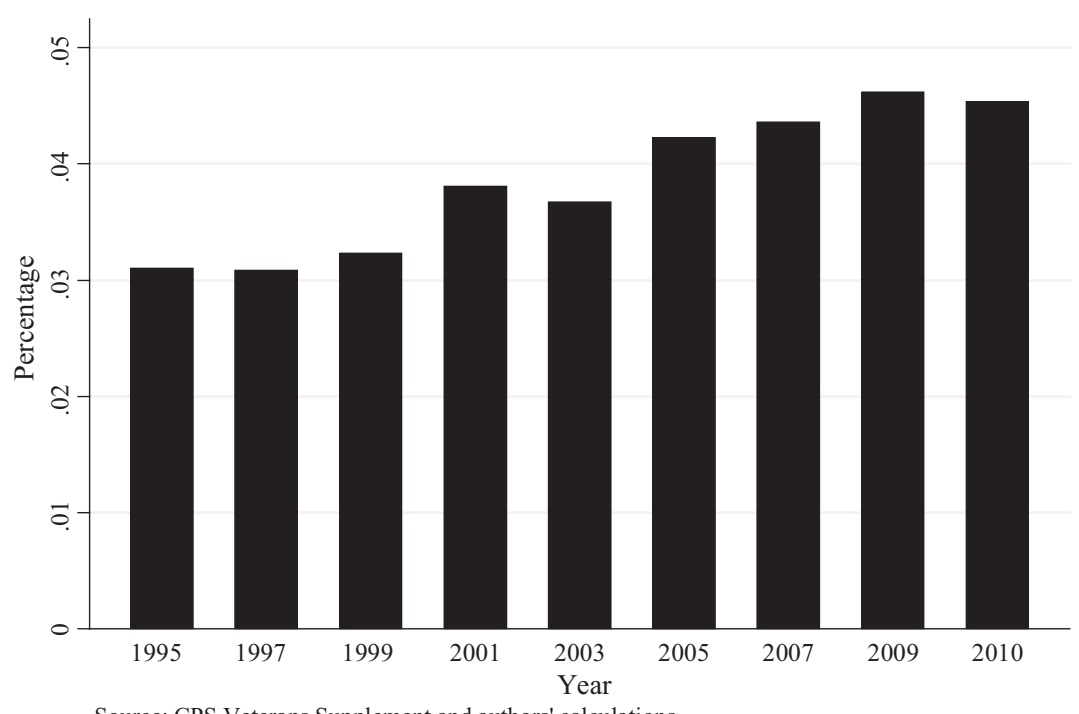

Source: CPS Veterans Supplement and authors' calculations

Fig. 2. Self-report of disability using ability to work question.

fore use the more direct self-report measure as our comparator because it is reasonably consistent with the "holistic" characterization in the labor force recode question and, because of relatively infrequent affirmative answers, provides a more conservative estimate of disability status.

Figure 3 displays the external reference point: administrative agency determinations of disability status. The overall trend created by the height of each bar mirrors almost identically the same in Fig. 2. However, the striking difference between the two graphs lies in scale.
By 2010, VA/DoD considered $15 \%$ of the CPS respondents to have a service-connected disability, whereas only $4.5 \%$ respondents themselves claimed a disability. Over the entire time period, $29 \%$ of self-reporters were considered disabled by the federal government, and about $9 \%$ of those classified as disabled by VA/DoD reported a disability themselves. Part of the latter disparity stems from the fact that persons with disabilities still can work; indeed, $42 \%$ of the positive agency determinations reported being employed in the previous week. Another $48 \%$ either were not working or were 


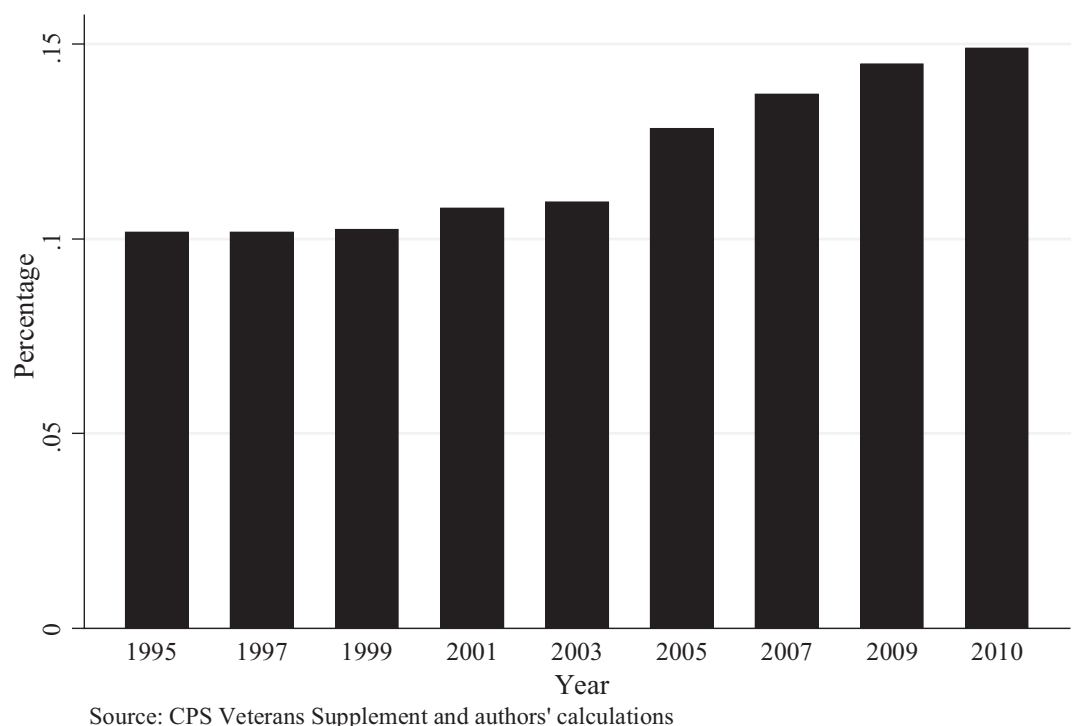

Fig. 3. VA/DoD disability determination.

retired. At the end of the day, a within-subjects examination (i.e., comparing variables for each respondent rather than across respondents) suggests that selfconsiderations of disability status are much less frequent than external decisions.

Figures 4 and 5 reveal perhaps the most important trends with respect to veterans' disabilities. In the former, we notice a secular increase in more severe mental and physical conditions among those with disabilities. For purposes of determining benefits eligibility, the VA or DoD assigns a disability rating to veterans with disabilities. In 2012, a $10 \%$ rating entitled a veteran to $\$ 127$ per month, while $70-100 \%$ ratings increased the monthly allotment (for veterans without children) to approximately $\$ 1300-\$ 2800$, respectively [21]. The CPS data do not reveal the specific disability rating but rather aggregate them into eight groups of differing ranges. We combined those categories further into "low," (0-29\%) "medium", (30-50\%) and "high" (51\%-100\%) designations.

The three lines in Fig. 4 correspond to those three ranges and represent the percentage of veterans with disabilities in each one. In 1995, about two-thirds of veterans with disabilities were considered to have relatively less severe conditions. Meanwhile the share of veterans with medium- and high-range disabilities grew, albeit at a slower rate. One obviously cannot attribute the steady decline of lower-rated disabilities through the early 2000s to deployments in Iraq and Afghanistan. Regardless of the cause, the clear trend indicates that, if service-connected disability
Table 2

Disability reports by veteran characteristics

\begin{tabular}{lcc}
\hline Variable & $\begin{array}{c}\text { Self- } \\
\text { reported }\end{array}$ & $\begin{array}{c}\text { VA/DoD } \\
\text { determined }\end{array}$ \\
\hline Male & $3.8 \%$ & $11.7 \%$ \\
Female & $3.3 \%$ & $13.4 \%$ \\
White & $3.5 \%$ & $11.4 \%$ \\
Non-white & $6.3 \%$ & $15.4 \%$ \\
Education & & \\
Less than high school & $7.3 \%$ & $11.8 \%$ \\
Less than a bachelor's degree & $4.1 \%$ & $11.8 \%$ \\
At least a bachelor's degree & $1.5 \%$ & $11.9 \%$ \\
\hline
\end{tabular}

Source: CPS Veterans Supplement and authors' calculations.

rates expand, discrepancies between self-identification and government determination will exacerbate any associated difficulty in targeting veterans most in need of treatment.

Figure 5 portrays the life-cycle, as it were, of service-connected disabilities using both the self-report and VA/DoD measures. Even though the two designations converge over time, the VA/DoD curve always lies above the self-report version, and significant convergence probably does not arise until 15-20 years after separation. One implication might be that younger veterans will obtain treatment at rates well below that which an independent determination would suggest and thus cut themselves off from available health treatment and employment training exactly when they need them most.

Further disaggregation of the data by observable characteristics uncovers other differences in disability reporting of likely relevance to policy discourse. Here, 


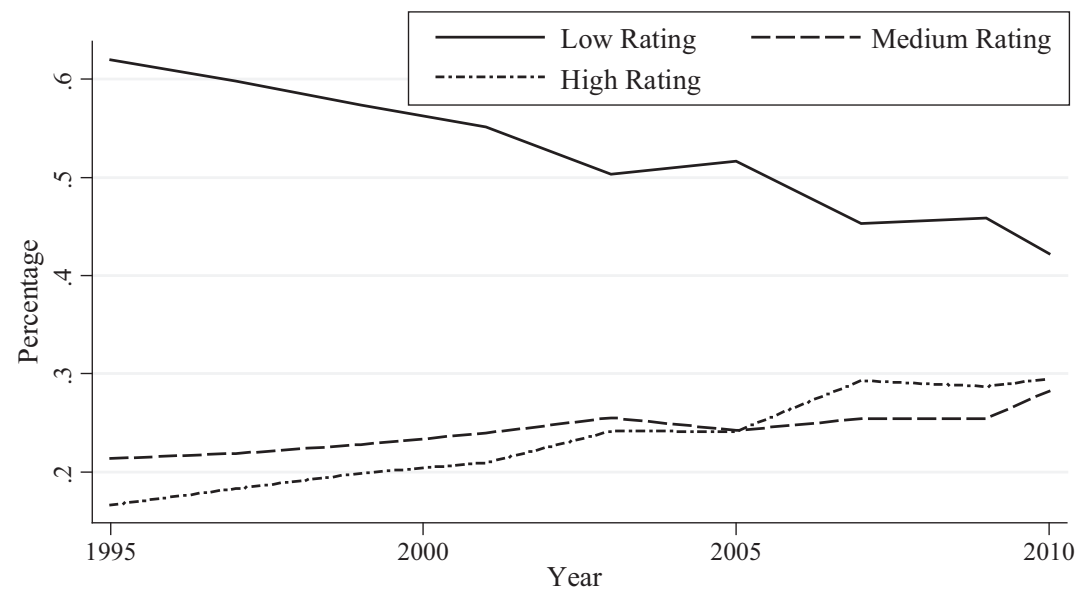

Source: CPS Veterans Supplement and authors' calculations

Fig. 4. VD/DoD disability rating percentages.

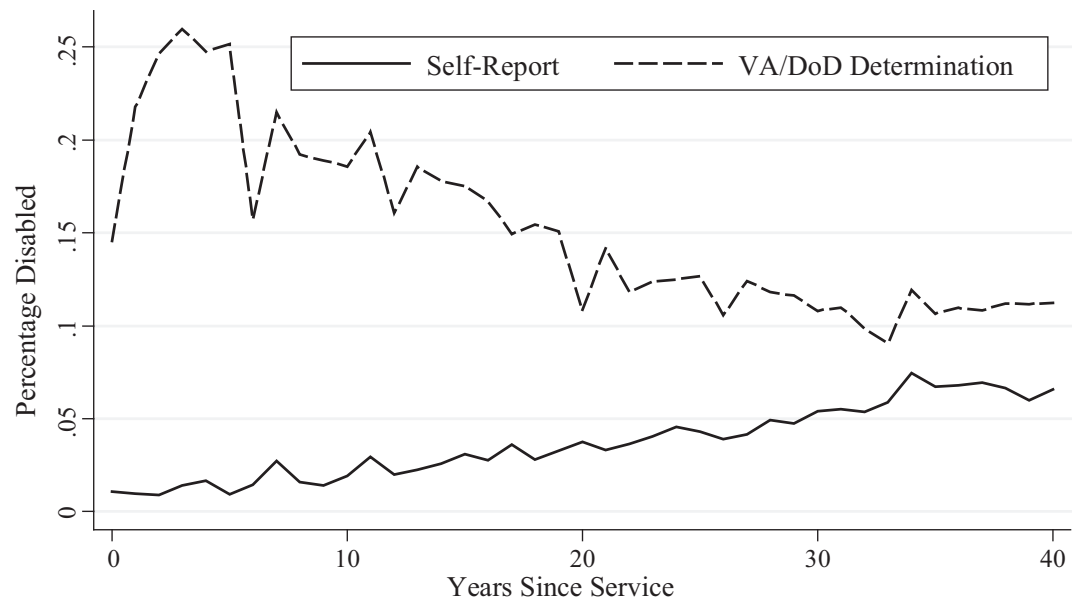

Source: CPS Veterans Supplement and authors' calculations

Fig. 5. Disability reports by year of separation from the armed forces.

we calculate the percentage of all veterans by characteristic reporting or having a disability reported across the entire time period 1995-2010, the results of which are reported in Table 2.

Large differences between self-reports and agency findings stand out immediately as does the higher variance of self-reported disability status by veteran characteristics relative to VA/DoD conclusions. However, both disability measures indicate that non-white veterans have service-connected disability rates around 3-4 percentage points higher than white veterans. Less educated veterans also tend to report having a disability more often, but educational background appears not to affect the likelihood of receiving a VA/DoD confirmation.
Of the nearly 11,000 veterans with a disability designated by the VA/DoD, just under 9800 of those observations also contain non-missing data on their agency disability rating. Combining those rating data with self-report information, the story becomes slightly more heartening. Specifically, the share of respondents self-reporting a disability is monotonically increasing in the severity of their underlying conditions. Although only $3.6 \%$ of the "low" disability veterans themselves report having one, the "medium" group rate climbs to $6 \%$, and the "high" category self-identifies as disabled $25.7 \%$ of the time. The optimistic inference is that a strong correlation exists between the externally perceived gravity of one's disability and the probability that the veteran also will consider him/herself disabled. 
Finally, we add some sense of statistical and practical significance regarding differentials in the propensity to report either disability measure. We focus only on the raw difference, i.e., without controlling for any other factors, because we will gain little additional purchase from complicating the empirical model with purely observational CPS data. Although nearly all of the veterans in the sample are men, we found a statistically significant, somewhat higher likelihood that they would self-report having a disability $(p<0.05)$ and a lower, more highly significant rate of VA/DoD reports $(p<0.01)$. Given our broader hypothesis regarding the correlation between service-connected norms about masculinity and strength, these results might be counterintuitive. Then again, these significance tests clearly are biased by our excluding a host of other correlated but unavailable variables. Both sexes, however, saw an increase in the rate of self- and governmentbased disability reports over the observation period (e.g., increases in the VA/DoD disability rate from $10.1 \%$ to $14.5 \%$ and $10.9 \%$ to $19.9 \%$ for men and women, respectively).

Examining trends with respect to educational background, veterans with at least a bachelor's degree were $3 \%$ less likely to report having a disability relative to those with at least a high school diploma but no college degree $(p<0.01)$; no similar distinction emerged in the VA/DoD reports. On the other hand, the clearest differentials materialize in connection with race. White veterans self-reported disabilities $3 \%$ less often than non-white veterans and reported an external determination at a $4 \%$ lower rate; both results are significant at the $1 \%$ level. Even more striking is the relative growth rate between 1995 and 2010 in disability reports. Whereas white veterans saw increases of less than 2 percentage points and about 5 percentage points for self-reports and government determinations, respectively, the same figures for non-white respondents are about 10.5 percentage points and a near tripling in VA/DoD reports (from $12.9 \%$ to $34.9 \%$ ). Escalations of this magnitude at least intimate that the incidence of and gap in service-connected disabilities between white and non-white veterans will merit consideration in any policy addressing disability underreporting.

\section{Discussion}

If the patterns observed in the data above are connected to a military culture that downplays, ignores, or even denigrates disability status, very troubling consequences for post-service employment are likely to follow. Recent indicators point to hopeful developments with respect to labor force reintegration; for example, the unemployment rate of veterans with disabilities was five percentage points greater than the general population of Americans with disabilities [22]. On the other hand, estimates disaggregated by theaters of service signify higher unemployment $(9.9 \%)$ and disability rates (26\%) among Iraq War veterans relative to all former service members $(6.7 \%$ and $14 \%$, respectively) [23]. Generally speaking, current empirical evidence [24] points to decreased economic opportunity for veterans with work-limiting disabilities.

As with population-wide public health and employment predicaments, policymakers might start with channeling purely financial resources toward disabled veterans. Recent research shows that even indirect funds, tax expenditures in the form of additional income credits, can improve the employment outlook of disabled veterans. One empirical examination suggests that new "tax credits generated a statistically significant 2 percentage point increase in employment. This impact translates to an additional 32,000 employed disabled veterans per year over 2007 and 2008" [25]. But pecuniary assistance will be of little long-term value if persistent unemployment is caused in part by unwillingness to acknowledge one's disability.

Based on the CPS data explored in this paper, we can at best surmise a hypothesis for a causal relationship between low disability self-identification and employment outcomes for U.S. veterans. Eligibility for programs such as the Vocational Rehabilitation and Employment Program administered by the Department of Veterans Affairs requires former service members to 1) have at least a $20 \%$ disability rating and 2) work with a counselor to determine whether they have employment-related disabilities. These mandates implicitly require acceptance of physical or mental conditions that affect one's ability to work, precisely the admission that the data find wanting. Many veterans surely need assistance with locating matches in the labor market that maximize use of their human capital. The success of this matching process, in turn, can mean the difference between gainful employment and a frustrated reintegration into society. When disability status interacts with this process, we hypothesize that reluctance to self-identify as disabled, driven in large part by a military culture that perceives disability as weakness, will hamper access to training programs and eventual employment. 
Although we do not delve more deeply into the normative backdrop of service, we believe that one positive step might include earlier VA/DoD rating assessments, i.e., before veterans apply for federal benefits. The evidence in Fig. 5 reinforces the time sensitivity of aligning self-perception of disability with an external, medical appraisal. A program that increased disability diagnosis rates among the veteran population would could lead to earlier convergence and eventually encourage new norms about disability in the armed forces. Providing near-universal evaluations might increase the demand for assistance, and the marginal benefit of more quickly transitioning veterans to civilian employment (thereby reducing outlays for disability and unemployment insurance) likely would exceed the marginal costs. Early detection might spur veterans to internalize more fully the realities of their mental and physical conditions and seek additional rehabilitation or training to prepare for workforce reentry.

\section{Conclusion}

This paper has documented the gap between selfperception and government determination of disability among American veterans. Based on national survey evidence, it is abundantly clear that returning veterans are far less likely than the VA or DoD to acknowledge a service-related disability. Military values that discount weakness likely contribute to this disconnect. Even without direct confirmation that links norms and perceptions, the data show surprisingly large differentials between internal and external assessments of disability status, some of which are exacerbated by race and differences in educational background. Improving disabled veterans' employment prospects will hinge on reforming government programs that identify and assist those with disabilities as much as overcoming stigmas.

Possible remedial steps include significant outreach to racial minorities and those with lower educational attainment. Those efforts should target barriers to selfacknowledgement about the presence of a servicerelated disability. Without full acceptance and awareness of their conditions, veterans will be reluctant to address them with the VA/DoD, even setting aside current issues with the processing of benefit claims. Ultimately, the administration and wounded warriors themselves owe a duty to the entire military community to change the perception of disability and improve employment outcomes.

\section{References}

[1] Gerber D, editor, Disabled veterans in history. Ann Arbor: University of Michigan Press, 2000.

[2] Cohen D. The war come home: Disabled veterans in Britain and Germany, 1914-1939. Berkley: University of California Press, 2001.

[3] Mor S. Between charity, welfare, and warfare: A disability legal studies analysis of privilege and neglect in Israeli disability policy. Yale J Law Hum. 2000; 18(2): 63.

[4] Moore A, Kornblet, S. Introduction, In: Moore A, Kornblet $S$, editors, Advancing the rights of persons with disabilities: A US-Iran dialogue on law, policy and advocacy. Washington DC: Stimson Center, 2011.

[5] Logue L, Blanck P. Race, ethnicity, and disability: veterans and benefits in post-Civil War America. Cambridge: Cambridge University Press, 2010.

[6] Logue L, Blanck P. Benefit of the doubt: African - American Civil War Veterans and Pensions. J Interdiscip Hist. 2008; 38(3): 377

[7] Buddin R, Kapur, K. RAND National Defense Research Institute. An analysis of military disability compensation [monograph online]. Santa Monica: RAND Corporation; 2005 [cited 2014 Feb 01]. Available from: http://www.rand.org/pubs/ monographs/MG369.html.

[8] Sherman N. Stoic warriors: The ancient philosophy behind the military mind. New York: Oxford University Press, 2005.

[9] Lunasco TK, Goodwin EA, Ozanian AJ, Loflin EM. One shotone kill: A culturally sensitive program for the warrior culture. Military Medicine. 2010; 175(7): 509-513.

[10] Hubbard A. A military-civilian coalition for disability rights. Miss Law J. 2006; 75(4): 975.

[11] Rudd MD. Soldiers need help with the emotional toll of war. USA Today. 2010 Mar 3: Sect. A:9.

[12] Laughlin C. She secures treatment for vets' invisible wounds. The Philadelphia Inquirer. 2012 Nov 28: Sect. C:1.

[13] Klocek JW. The physical and psychological impact of your injury and disability. In: Ainspan ND, Penk WE, editors. Returning wars' wounded, injured, and ill: A reference handbook. Praeger Security International, 2008.

[14] Nash WP, Silva C, Litz B. The historic origins of military and veteran mental health stigma and the stress injury model as a means to reduce it. Psychiatr Ann. 2009; 39(8): 789-794.

[15] Hoge CW, Castro CA, Messner SC, McGurk D, Cotting DI, Koffman RL. Combat duty in Iraq and Afghanistan, mental health problems, and barriers to care. N Eng J Med. 2004; 351(1): 13-22.

[16] Krieger LM, Landhuis E. Iraq, Afghanistan leave invisible wounds on soldiers: fewer than half of returnees needing help got it, study shows. San Jose Mercury News. 2004 July 1: Sect. A: 3 .

[17] Musgrave J. Veterans face enemy at home: denial, posttraumatic stress disorder. Palm Beach Post. 2009 May 17: Sect. A: 1.

[18] Acemoglu D, Angrist JD, Consequences of employment protection? The case of the Americans with Disabilities Act. J Polit Econ. 2001; 109(5): 915-957.

[19] Jolls C, Prescott JJ. Disaggregating employment protection: the case of disability discrimination. NBER Work Pap Ser. 2004 Sept; No. 10740.

[20] Donohue JJ, Stein MA, Griffin CL, Becker S. Assessing postADA employment: Some econometric evidence and policy considerations. J Empir Leg Stud. 2011; 8(3): 477-503. 
[21] United States. Federal benefits for veterans, dependents and survivors [Internet]. Dept. of Veterans Affairs (US). Chapter 2, Disability compensation. [cited 2014 Feb 01] Available from: http://www.va.gov/opa/publications/benefits_book/ben efits_chap02.asp.

[22] Tennant J. Disability, employment, and income: are Iraq/ Afghanistan-era U.S. veterans unique?. Mon Labor Rev. 2012 Aug; 135(8): 3-10.

[23] Collins B, Bradley DH, Dortch C, Kapp L, Scott C. Employ- ment for veterans: trends and programs, Congressional Research Service (US); 2012 Oct. 17 p. Report No.: R47290.

[24] London, AS., Heflin CM, Wilmoth JM. Work-related disability, veteran status, and poverty: implications for family wellbeing. J Poverty. 2011; 15(3): 330-349.

[25] Heaton P. The effects of hiring tax credits on employment of disabled veterans, Santa Monica: RAND Corporation; 2012. 24 p. Report No.:OP-366-OSD. Disabled Veterans, RAND Corporation 2012. 